\title{
8. Über die Invarianten endlicher Gruppen linearer Substitutionen im Körper der Charakteristik p.
}

\author{
Von Kenjiro SHoda. \\ (Eingegangen am 22. April, 1940.)
}

In der vorliegenden Note soll der Endlichkeitssatz für die Invarianten der endlichen Gruppen (ganzer oder) gebrochener linearer Substitutionen im Körper beliebiger Charakteristik bewiesen werden. In der Literatur ist der Satz nur im Fall der Charakteristik Null angegeben. ${ }^{\left({ }^{1}\right)}$ Nach dieser Ausdehnung des Satzes kann man die Brauersche Theorie $\left({ }^{2}\right)$ des Kleinschen Formenproblems in den Fall der Charakteristik $p$ übertragen.

1. Den Ausgangspunkt bildet der Endlichkeitssatz für Permutationsgruppen. Es sei $k$ ein beliebiger Körper. Sind $s_{1}, \ldots, s_{n}$ die elementaren symmetrischen Funktionen der $n$ Variablen $x_{1}, \ldots, x_{n}$, so ist der aus den rationalen Funktionen von den $x$ mit Koeffizienten aus $k$ bestehende Körper $k(x)=k\left(x_{1}, \ldots, x_{n}\right)$ ein separabler galoisscher Erweiterungskörper von $k(s)=k\left(s_{1}, \ldots, s_{n}\right)$, dessen galoissche Gruppe die symmetrische Gruppe der $n$ Variablen $x_{i}$ ist. Der Polynombereich $k[x]=k\left[x_{1}, \ldots x_{n}\right]$ ist dann ein endlicher $k[s]$-Modul, da jedes Polynom von den $x_{i}$ sich nach der Gleichung

$$
x^{n}-s_{1} x^{n-1}+\cdots+(-1)^{n} s_{n}=0
$$

reduzieren lässt. In $k[s]$ gilt aber bekanntlich der Basissatz, weil die $n$ symmetrischen Funktionen $s_{i}$ algebraisch unabhängig, also $k[s]$ zu $k[x]$ isomorph ist. Daher kann man den Endlichkeittssatz für Permutationsgruppen folgendermassen formulieren.

Hilfssatz. Jeder Zwischenring von $k[s]$ und $k[x]$ ist ein endlicher $k[s]-$ Modul.

2. Wir werden nun den Endlichkeitssatz fưr endliche Kollineationsgruppen aus dem eben bewiesenen Hilfssatz ableiten. Da wir nur den

(1) Vgl. H. Weber, Lehrbuch der Algebra, 2. Aufl. §57. E. Noether, Der Endlichkeitssatz der Invarianten endlicher Gruppen, Math. Ann., 77 (1916), 89-92. R. Brauer, Über die Kleinsche Theorie der algebraischen Gleichungen, Math. Ann., 110 (1934), 473-500. Im Fall der Charakteristik $p$ ist der Satz nur für ganze lineare Substitutionsgruppen bewiesen. Vgl. E. Noether, Der Endlichkeitssatz der Invarianten endlicher linearer Gruppen der Charakteristik $p$, Göttinger Nachr., (1926), 28-35.

(2) R. Brauer, a.a.O. 
von R. Brauer( $\left.{ }^{3}\right)$ vorgezeigten Weg etwas $\mathrm{zu}$ modifizieren brauchen, behalten wir hier auch seine Bezeichnungen bei, die wir der Vollständigkeit halber wieder angeben werden.

Eine Kollineation

$$
x_{k}=\frac{a_{k 0}+a_{k 1} y_{1}+\cdots+a_{k n} y_{n}}{a_{c 0}+a_{01} y_{1}+\cdots+a_{0 n} y_{n}}
$$

bezeichnen wir mit $x=C(y)$. Setzt man

$$
\left(x_{k}\right)_{C}=\frac{a_{k 0}+a_{k 1} x_{1}+\cdots+a_{k n} x_{n}}{a_{00}+a_{01} x_{1}+\cdots+a_{0 n} x_{n}}
$$

und für eine rationale Funktion $f$

$$
\left.f_{C}\left(x_{1}, \ldots, x_{n}\right)=f\left(\left(x_{1}\right)_{C}, \ldots, x_{n}\right)_{C}\right) \text {, }
$$

so wird die Invariante durch $f=f_{C}$ definiert. Es gilt ferner im allgemeinen

$$
\left(f c_{1}\right)_{C_{2}}=f_{C_{1} C_{2}} .
$$

Es sei nun eine endliche Gruppe der Kollineationen $C$ vorgegeben. Ist $J=\frac{A}{B} \neq 0$ eine Invariante, wo $A$ und $B$ teilerfremde Polynome in den $x_{i}$ etwa von den Graden $a$ und $b, a \geq b$, sind, so erkennt man leicht

$$
\begin{aligned}
& A_{C}=A\left(\left(x_{1}\right)_{C}, \ldots,\left(x_{n}\right)_{C}\right)=\frac{h_{C} A\left(x_{1}, \ldots, x_{n}\right)}{z_{C}^{a}}, \\
& B_{C}=B\left(\left(x_{1}\right)_{C}, \ldots,\left(x_{n}\right)_{C}\right)=\frac{h_{C} B\left(x_{1}, \ldots x_{n}\right)}{z_{C}^{a}},
\end{aligned}
$$

wo $z_{C}$ der Nenner der Kollineation $C$ ist. Ist die Charakteristik von $k$ gleich $p$ und ist die Ordnung $h$ der Gruppe (S) durch $p^{r}$ genau teilbar, $h=p^{r} q,(p, q)=1$, so kann man (3) nach einer Sylowgruppe $\subseteq$ von der Ordnung $p^{r}$ zerlegen :

$$
\left(\mathfrak{S}=\subseteq P_{1}+\subseteq P_{2}+\cdots+\subseteq P_{q}\right. \text {. }
$$

Dann ist

$$
J^{p^{r}}=\frac{1}{q} \sum_{i=1}^{q} \operatorname{II}_{C \in P_{i}} J_{C}=\frac{1}{q} \sum_{i=1}^{q} \frac{\operatorname{II}_{C \in P_{i}} A\left(\left(x_{1}\right)_{C}, \ldots,\left(x_{n}\right)_{C}\right)}{\prod_{C \in \subseteq P_{i}} B\left(\left(x_{1}\right)_{C}, \ldots,\left(x_{n}\right)_{C}\right)} .
$$

(3) R. Brauer, a.a. O. $\$ 2$. 
Hieraus folgt

$$
\begin{aligned}
J^{p^{r}}=\frac{Z}{N}, \quad N & =q \operatorname{II~}_{C \in \mathbb{G}} B\left(\left(x_{1}\right)_{C}, \ldots,\left(x_{n}\right)_{C}\right) \\
Z & =\sum_{i=1}^{q} \prod_{D \in \mathbb{E}_{P}} A\left(\left(x_{1}\right)_{D}, \ldots,\left(x_{n}\right)_{D}\right) \operatorname{II}_{\substack{C \in \mathbb{E}_{i} \\
i \neq j}} B\left(\left(x_{1}\right)_{C}, \ldots,\left(x_{n}\right)_{C}\right)
\end{aligned}
$$

Ist die Charakteristik von $k$ gleich Null, so hat man nur $r=0$ zu setzen. Dies ist gerade der von R. Brauer betrachtete Fall. Bezeichnet man nun die hn Grössen $\left(x_{i}\right)_{C}$ in irgendeiner Reihenfolge mit $t_{1}, \ldots, t_{h n}$, so erhält man eine Permutation von den $t_{i}$, wenn man $\left(x_{i}\right)$ durch $\left(x_{i}\right)_{C}$ ersetzt. Dadurch erhält man eine zu (5) isomorphe Fermutationsgruppe (5*. $N$ unn $Z$ sind, aufgefasst als Polynome in den $t_{i}$, Invariante von (3). Nach dem Hilfssatz gibt es endlich viele Invarianten $J_{1}, \ldots, J_{k}$ so, daß jede ganze Invariante für $\left(3^{*}\right.$ aich als Polynom in den $J_{i}$ 'darstellen lääst :

$$
J^{p^{r}}=\frac{\phi\left(J, \ldots, J_{k}\right)}{\Psi\left(J_{1}, \ldots, J_{k}\right)}
$$

Das gilt natürlich auch, wenn man die $t_{i}$ wieder in ihrer ursprünglichen Bedeutung nimmt. Ist $r=0$, so gilt daher der Endlichkeitssatz für (5).

Wir nehmen jetzt an, daß der Grundkörper vollkomen ist. Ist die Charakteristik von $k$ gleich $p$, so sieht man leicht, daß der Invariantenkörper für (S) ein Zwischenkörper von $k\left(J_{1}, \ldots, J_{k}\right)$ und seine endliche Erweiterung $k\left(p^{r} / J_{1}, \ldots, \sqrt[p]{\delta_{k}}\right)$ ist. Also ist der Invariantenkörper eine endliche Erweiterung von $k\left(J_{1}, \ldots, J_{l}\right)$. Damit ist also gezeigt, daß der Invariantenkörper durch endlich vielen Invarianten erzeugt wird. Man kann ferner ersichtlich annehmen, daß der Invariantenkörper durch Adjunktion der Invarianten $J$ von der Gestalt

$$
J^{p^{r}}=\Phi\left(J_{1}, \ldots, J_{k}\right)
$$

aus $k\left(J_{1}, \ldots, J_{k}\right)$ entsteht, also, daß der Nenner von $J$, wie $J_{i}$, ein Potenzprodukt von den $z_{C}$ ist. Zusammenfassend erhält man den Endlichkeitssatz für endliche Gruppe (S) der Kollineationen in der folgenden Form :

Satz 1. Jede rationale Invariante für (S) lässt sich als rationale Funktion der endlich vielen Grundinvarianten $J_{i}$ darstellen. Als den Nenner von $J_{i}$ kann man ein Potenzprodukt der Nenner der Kollineationen annehmen.

3. Für eine endliche Gruppe (S) ganzer linearer Substitutionen kann man analog vorgehen und dadurch erhält man wieder die formel 


$$
J^{p^{r}}=\Phi\left(J_{1}, \ldots, J_{k}\right),
$$

wo $J, J_{1}, \ldots, J_{k}$ jetzt Polynome aus $k[x]$ sind. Ist $k$ vollkommen, so ist $J$ ein Polynom von den $p^{r}, \bar{J}$. Also ist der Invariantenbereich für $\mathbb{S}$ in einem endlichen $k\left[J_{1}, \ldots, J_{k}\right]$-Modul enthalten. Fasst man $k\left[J_{1}, \ldots, J_{k}\right]$ wieder als Polynombereich $T$ von den $t_{i}$ auf, so ist $T$ ein endlicher $k[s]$ -Modul, also gilt nach dem Hilfssatz der Basissatz in $T$. Der daraus durch Speziallisierung von den $t_{i}$ entstehende Bereich $k\left[J_{1}, \ldots, J_{k}\right]$ ist zu $T$ homomorph, also gilt in $k\left[J_{1}, \ldots, J_{k}\right]$ auch der Basissatz, Daher ist der Invariantenbereich für $\$ \$$ ein endlicher $k\left[J_{1}, \ldots, k_{k}\right]$-Modul.

Satz 2. Jede ganze rationale Invariante für (5) lässt sich als ganze rationale Funktion der endlich vielen Grundinvarianten darstellen.

4. Die obigen beiden Sätze gelten im allgemeinen auch, wenn der Grundkörper $k$ unvollkommen ist. Das ist eine unmittelbare Folgerung von

Satz 3. Gilt der Endlichkeitssatz einer ganzen oder gebrochenen Substitutionsgruppe in einem algebraischen Erweiterungskörper von $k$, so gilt er auch im Grundkörper $k$.

Es sei nämlich $k^{\prime}$ eine algebraische Erweiterung von $k$. Sind $I_{1}, \ldots, I_{m}$ die Grundinvarianten füe (\$) mit Koeffizienten aus $k^{\prime}$, so sind die Koeffizienten von den $I_{i}$ in einem endlichen Erweiterung von $k$ enthalten. Daher nehmen wir an, daß $k^{\prime}$ endlich bezüglich $k$ ist, Sind $\omega_{1}, \ldots, \omega_{N}$ die Körperbasis von $k^{\prime}$, so lässt sich $I_{i}$ eindeutig in der Form

$$
I_{i}=J_{i}^{(1)} \omega_{1}+\cdots+J_{i}^{(N)} \omega_{N}
$$

darstellen, wo die $J_{i}^{(1)}$ in $k[x]$ liegen. Dann sind die $J_{i}^{(j)}$ auch Invarianten für (5) und der Invariantenbereich (Invariantenkörper) wird durch den $J_{i}^{(j)}$ erzeugt. Ist (53 eine Kollineationsgruppe und ist der Nenner von $I$ eine Potenzprodukt der Nenner der Kollineationen, so gilt dasselbe auch für den Nenner von J. Daher kann man die in Satz 1 bzw. 2 angegebenen Grundinvarianten stets in $k[x]$ annehmen. Ist $F$ eine Invariante aus $k(x)$, so lääst sich $F$ in der Form

$$
F=F_{1} \omega_{1}+\cdots+F_{N} \omega_{N}
$$

darstellen, wo die $F_{i}$ Funktionen von den Grundinvarianten $J_{i}$ sind. Wenn man nun $\omega_{1}=1$ annimmt, wie immer möglich ist, so ergibt sich hieraus $F=F_{1}$, also ist $F$ eine Funktion von den $J_{i}$ mit Koeffizienten aus $k$. Damit ist der Satz bewiesen.

5. Wir werden nun die Invarianten einer endlichen Gruppe (S) linearer Substitutionen als eine Anwendung der Darstellungstheorie untersuchen. Wir beweisen zunächst $\left({ }^{4}\right)$

(4) Diesen Satz verdanke ich T. Nakayama. Zum Beweis benützt er gewisse Struktursätze über Algebren. 
Satz 4. Es sei $\mathfrak{A}$ eine Algebra, $M(\alpha)$ eine Darstellung von $\mathfrak{A}$ durch Matrizen, L(a) eine linke reguläre Darstellung von $\mathfrak{A}$. Für hinreichend grosses $m$ enthält die Darstellung

$$
m L(a)=\left(\begin{array}{llll}
L(a) & & & \\
& L(a) & & \\
& & \ddots & \\
& & \cdot(a)
\end{array}\right)
$$

die Darstellung $M(a)$ als (nicht notwendig direkten) Bestandteil:

$$
m L(a) \simeq\left(\begin{array}{cc}
M(a) & 0 \\
* & *
\end{array}\right) .
$$

Ist $m$ der linke Darstellungsmodul für $M(a)$, so kann man ohne wesentliche Beschränkung der Allgemeinheit annehmen, daß $\mathfrak{U} \mathfrak{m}=\mathfrak{m}$ ist. Ist der Rank von $\mathfrak{A}$ bzw. m gleich $h$ bzw. $m$, so nehmen wir hm linear unabhängige Elemente $u_{\alpha}^{(\lambda)}, \kappa=1, \ldots, h, \lambda=1, \ldots, m$, an so, daß die $h$ Elemente $u_{1}^{(\lambda)}, \ldots, u_{h}^{(\lambda)}$ einen zu $\mathfrak{H}$ operatorisomorphen Darstellungsmodul bilden. Dann vermittelt der aus den sämtlichen $u_{x}^{(\lambda)}$ gebildete Darstellungsmodul $\mathfrak{u}$ die Darstellung $m L(a)$. Ist $a_{x}$ das $u_{x}^{(\lambda)}$ zugeordnete Element aus $\mathfrak{A}$, so erhält man eine homomorphe Abbildung von $\mathfrak{U}$ auf $\mathfrak{A}_{\mathfrak{m}}=\mathfrak{m}$, wenn man $u_{*}^{(\lambda)}$ auf $a_{\kappa} \varepsilon_{\lambda}$ abbildet. Dabei bedeutet das System $\varepsilon_{1}, \ldots, \varepsilon_{m}$ ein Basis von $m$. Das besagt in der Darstellungstheorie nichts anderes als den Satz 4 .

Wir betrachten nun ganze Invarianten einer Gruppe (S) ganzer linearer Substitutionen

$$
\left(x_{1}, \ldots, x_{n}\right)=\left(y_{1}, \ldots, y_{n}\right) A
$$

mit Matrizen $A$ des Grades $n$. Es sei $(5$ reduzibel :

$$
\begin{aligned}
\left(x_{1}, \ldots, x_{n}\right) & =\left(X_{1}, \ldots, X_{n}\right) P \\
\left(X_{1}, \ldots, X_{n}\right) & =\left(Y_{1}, \ldots, Y_{n}\right)\left(\begin{array}{cc}
A_{1} & 0 \\
B & A_{2}
\end{array}\right)
\end{aligned}
$$

wo der Grad von $A_{1}$ bzw. $A_{2}$ gleich $n_{1}$ bzw. $n_{2}$ ist. Ist $J$ eine Invariante für $\left(\mathcal{S}\right.$, so erhält man eine Invariante $J_{1}$ für die aus den Substitutionen

$$
\left(X_{1}, \ldots, X_{n_{1}}\right)=\left(Y_{1}, \ldots, Y_{n_{1}}\right) A_{1}
$$

bestehende Gruppe $\mathbb{G}_{1}$, wenn man in $J$ alle $Y=0$ für $i>n_{1}$ setzt. Es ist nämlich

$$
J \equiv J_{1} \quad\left(\bmod Y_{n_{1}+1}, \ldots, Y_{n}\right)
$$


Das Ideal $\left(Y_{n_{1}+1}, \ldots, Y_{n}\right)$ in $k\left[Y_{1}, \ldots, Y_{n}\right]$ wird durch die Substitutionen aus $\left(\mathcal{G}\right.$ in sich übergeführt. Daher werden $Y_{1}, Y_{2}, \ldots, Y_{n_{1}}$, modulo dem Ideal betrachtet, unter ihnen transformiert:

$$
\left(X_{1}, \ldots, X_{n_{1}}\right) \equiv\left(Y_{1}, \ldots, Y_{n_{1}}\right) A_{1} .
$$

Aus der Invarianteneigenschaft von $J$ für $\$$ folgt also unmittelbar die von $J_{1}$ für $\mathfrak{G}_{1}$. Die sämtlichen Polynome, die, modulo dem Ideal $\left(Y_{n_{1}+1}, \ldots, Y_{n}\right)$ betrachtet, Invarianten für $\&$ sind, bilden einen den Invariantenbereich für (S) enthaltenden Bereich $B$. Setzt man in einem Polynom aus $B Y_{n_{1+1}}=\ldots=Y_{n}=0$, so erhält man eine Invariante für $\left(\mathfrak{S}_{1}\right.$; und jede Invariante für $\mathbb{S}_{1}$ kann man dadurch erhalten.

Satz 5. Sind der Invariantenbereich für \&s und alle Oberbereich endlich, so ist der Invariantenbereich für einem Bestandteil (3. $_{1}$ auch endlich.

Ist nun (S) eine endliche Gruppe, so ist ihre reguläre Darstellung eine Permutationsgruppe, also ist $\mathbb{S}_{1}$ in einer Permutationsgruppe $\mathbb{S}$ als Bestandteil enthalten Daher folgt der Endlichkeitssatz für $\mathbb{G}_{1}$ unmittelbar aus Sätze 4 und 5 .

6. Jede Kollineation wird bekanntlich durch eine ganze lineare Substitution bestimmt. Dadurch kann man jede Kollineationsgruppe $\mathfrak{S}$ von $n$ Variablen durch eine ganze Substitutionsgruppe (\$) von $n+1$ Variablen bestimmen. Wir untersuchen nun die Beziehungen zwischen den Invarianten für $\mathfrak{5}$ und $\left(\mathfrak{S}\right.$. Ist $F_{\mathfrak{g}}=F\left(x_{1}, \ldots, x_{n}\right)$ eine Invariante für $\mathfrak{S}$, so ist $F_{B}=F\left(\frac{x_{1}}{x_{0}}, \ldots, \frac{x_{n}}{x_{0}}\right)$ eine Invariante für $(\mathcal{G}$. Es ist dann $F_{\mathscr{G}}=\frac{A}{B}$ mit ganzen homogenen $A$ und $B$ vom gleichen Grad, etwa $a$. Umgekehrt bestimmt solche Funktion eine Invariante für $55^{\circ}$.

Satz 6. Der Invariantenbereich für 5 ist endlich, wenn der Invariantenbereich der zugehörigen Gruppe (S) enalich ist.

Es sei namlich der Invariantenbereich für (5) endlich. Ist.

$$
F_{G}=\frac{A}{B}=\frac{\Phi\left(J_{1}, \ldots, J_{k}\right)}{\Psi\left(J_{1}, \ldots, J_{k}\right)}
$$

mit den Grundinvarianten $J_{1}, \ldots, J_{k}$ für (5). Der Grad von $J_{i}$ sei gleich $m_{i}$. Ist $d$ der grösste gemeinsame Teiler von den $m_{i}$,

so setze $\operatorname{man}\left({ }^{5}\right)$

$$
\sum_{i=1}^{k} l_{i} m_{i}=d, \quad m_{i}=n_{i} d,
$$

$$
J=\prod_{i=1}^{k} J_{i}^{l_{i}}
$$

(5) Diese Konstruktion von $\mathrm{J}$ verdanke ich $\mathrm{K}$. Asano. 
Dann ist der Grad von $J$ gleich $d$ und der von $\frac{J_{i}}{J^{n}}$ gleich Null. Also bestimmt $\frac{J_{j}}{J^{n_{i}}}$ eine Invariante für $\xi_{a}$. Divisiert man den Zähler und den Nenner durch $J^{\frac{a}{d}}$, so erkennt man, daß Frationale Funktion von den $\frac{J_{i}}{J^{n_{i}}}$ ist. Damit ist der Satz bewiesen.

Ist (3) eine endliche Gruppe, so kann man (3) als endliche Gruppe annehmen, da das Faktorensystem in einem gewissen Wrweiterungskörper einem aus Einhoitswurzeln bestehenden assoziiert ist. Die Bestimmung der Grundinvarianten für endliche Kollineationsgruppe ist also eine Folgerung derselben für endliche ganze Substitutionsgruppe.

Mathematisches Institut

Kaiserliche Universität zu Osaka. 